\title{
Quantifying and Understanding the Differences in Visual Activities with Contrast Subsequences
}

\author{
$\mathrm{Yu} \mathrm{Li}$ \\ Department of Computer Science \\ University of Missouri \\ Columbia, Missouri \\ ylrbc@mail.missouri.edu
}

\author{
Carla Allen \\ School of Health Professions \\ University of Missouri \\ Columbia, Missouri \\ allencar@health.missouri.edu
}

\author{
Chi-Ren Shyu \\ Informatics Institute \\ University of Missouri \\ Columbia, Missouri \\ shyuc@missouri.edu
}

\begin{abstract}
Understanding differences and similarities between scanpaths has been one of the primary goals for eye tracking research. Sequences of areas of interest mapped from fixations are a major focus for many analytic techniques since these sequences directly relate to the semantic meaning of the visual input. Many studies analyze complete sequences while overlooking the micro-transitions in subsequences. In this paper, we propose a method which extracts subsequences as features and finds contrasting patterns between different viewer groups. The contrast patterns help domain experts to quantify variations between visual activities and understand reasoning processes for complex visual tasks. Experiments were conducted with 39 expert and novice radiographers using nine radiology images corresponding to nine levels of task complexity. Identified contrast patterns, validated by an expert, prove that the method effectively reveals visual reasoning processes that are otherwise hidden.
\end{abstract}

\section{CCS CONCEPTS}

- Information systems $\rightarrow$ Data mining; • Human-centered computing $\rightarrow$ User models; $\bullet$ Applied computing $\rightarrow$ Psychology.

\section{KEYWORDS}

eye movement, scanpath, pattern analysis, contrast mining, diagnostic reasoning, radiographer

\section{ACM Reference Format:}

Yu Li, Carla Allen, and Chi-Ren Shyu. 2019. Quantifying and Understanding the Differences in Visual Activities with Contrast Subsequences. In 2019 Symposium on Eye Tracking Research and Applications (ETRA '19), fune 25-28, 2019, Denver, CO, USA. ACM, New York, NY, USA, 5 pages. https: //doi.org/10.1145/3314111.3319842

\section{INTRODUCTION}

One of the primary goals for scanpath analysis is understanding the reasoning processes behind the visual activities. Over the years, various methods have been proposed using pupillometry [Appel et al.

Permission to make digital or hard copies of all or part of this work for personal or classroom use is granted without fee provided that copies are not made or distributed for profit or commercial advantage and that copies bear this notice and the full citation on the first page. Copyrights for components of this work owned by others than the author(s) must be honored. Abstracting with credit is permitted. To copy otherwise, or republish, to post on servers or to redistribute to lists, requires prior specific permission and/or a fee. Request permissions from permissions@acm.org.

ETRA '19, June 25-28, 2019, Denver, CO, USA

(C) 2019 Copyright held by the owner/author(s). Publication rights licensed to ACM. ACM ISBN 978-1-4503-6709-7/19/06 . \$15.00

https://doi.org/10.1145/3314111.3319842
2018; Chen and Epps 2014], fixation and saccade characteristics [Le Meur and Coutrot 2016; Mills et al. 2011], geometrical trajectory properties [Haass et al. 2016; Jarodzka et al. 2010], spatial distribution of fixations [Borji et al. 2013; Lao et al. 2017], or sequences of annotated areas of interest (AOI) [Cristino et al. 2010; Eraslan et al. 2014]. Among these methods, some are suitable for certain analysis but lack critical semantic or temporal features to investigate how visual tasks are accomplished. Analyzing annotated AOI sequences ties most closely to the visual task and reasoning processes since it directly corresponds to the sequence of visual information and potentially captures the semantic meaning of the visual area.

However, many studies that use AOI sequence process entire sequence as a single feature. This is partially due to the lack of complexity of the visual tasks, such as timed free viewing [Eraslan et al. 2014], object search [Cristino et al. 2010] or simple questions [Chuk et al. 2017], which typically yield short scanpaths containing limited number of transitions between AOIs. For complex visual tasks, such as radiological or pathological diagnosis, the differences in eye movements can be significant with varying lengths [Cao et al. 2016; Gegenfurtner et al. 2011], even within the same expertise group [Shin et al. 2017]. Analysis techniques that rely on high within-group consistency or constant-length scanpaths would not be applicable to these situations.

Additionally, only limited or coarse sequence information is captured while the actual detailed transitions are overlooked in some studies. Studies employing probabilistic methods commonly use a transition matrix to model the connection between AOIs. But since first order Markovian property is almost always assumed [Chuk et al. 2014; Simola et al. 2008], the transition matrix only accounts for the next immediate transition. Machine learning methods, e.g. support vector machine [R. Tavakoli et al. 2015] or naïve Bayes [Raptis et al. 2017], are capable of classifying scanpaths to viewer groups and predicting visual tasks. The results or model produced from these probabilistic models are not always intuitive, complicating the interpretation of underlying visual reasoning processes.

Many methods transfer the AOI sequence into a string to retain complete transition information while producing intuitive result sequences. eyePattern [West et al. 2006] and SubsMatch [Kübler et al. 2014, 2017] use subsequences, but the analysis process allows no flexibility in subsequences, which makes it susceptible to noises. Methods that find the shortest common supersequences [Räihä 2010] and subsequences [Eraslan et al. 2014; Goldberg and Helfman 2010; Mast and Burmester 2011] consider the flexibility in scanpaths. However, the common patterns extracted by superimposing the AOI sequences may not exist in any of the original scanpaths and do not reflect the actual visual reasoning processes. For example, 
"AB" can be a result from many scanpaths starting with "A" and ending with "B", which could be misleading for some applications.

The present study aims to analyze scanpaths produced by complex visual tasks and extract group-specific visual activities from the AOI sequences of different lengths. The proposed method uses subsequences of the original AOI sequence to find frequent contrasting patterns between different viewer groups. Hierarchical clustering is deployed on the contrast patterns to find representative visual reasoning strategies. The process allows some flexibility so that the minor variances will not contaminate the results while the identified patterns are still based on actual subsequences and reveal the underlying visual reasoning processes.

\section{METHOD}

The proposed method uses contrast mining [Dong and Bailey 2012] on the subsequences of AOI sequences to find contrast subsequences that are the distinctive visual activities appearing frequently in one group but not in the others. These subsequences reflect the viewers' visual activities and reveal the visual reasoning processes for the task. They are organized into hierarchical clusters according to their similarities and the flat clusters at different heights show the correlation between AOIs and different levels of tasks.

\subsection{Temporal Binning and Subsequence Extraction}

Our method assumes that the fixation coordinates have been mapped to predefined AOIs. Let $L=\left\{l_{1}, l_{2}, l_{3} \cdots\right\}$ be a set of AOI labels. Let $S=s_{1} s_{2} \cdots s_{n}$ be a sequence of AOI labels of length $n, s_{i} \in L$ is the $i^{t h}$ label in the sequence. $S_{i, j}=s_{i} s_{i+1} \cdots s_{j}$ denotes the subsequence of $S$ from position $i$ to $j$.

To preserve temporal information, binning is applied to the mapped AOI sequences. Longer fixation in one AOI will be represented as a series of self-repeating labels. For example, $150 \mathrm{~ms}$ of $s_{i}=l_{i}$ in the original sequence will be represented as $s_{i} s_{i+1} s_{i+2}$ with a bin size of $50 \mathrm{~ms}$, where $s_{i}=s_{i+1}=s_{i+2}=l_{i}$.

Subsequences are extracted with a varying length sliding window method. The minimum and maximum lengths of the window are defined according to the visual task. To compensate for the small variation in AOI sequence which is caused by microsaccades, occasional inaccurate recording or simply stray fixations, the method allows a small number of differences for two subsequences to be considered similar. The similarity measurement for two subsequences $S_{i, j}$ and $S_{x, y}$ is defined as the Levenshtein distance $d\left(S_{i, j}, S_{x, y}\right)=\operatorname{lev}_{S_{i, j}, S_{x, y}}\left(\left|S_{i, j}\right|,\left|S_{x, y}\right|\right)=D_{x y}^{i j}$. Two subsequences $S_{i, j}$ and $S_{x, y}$ are considered as approximate subsequences if $d\left(S_{i, j}, S_{x, y}\right) \leq \theta$, where $\theta$ is a pre-defined edit distance threshold. With this threshold, the sliding window will have a gap of $\theta$ between two beginnings and the window length will be $\theta$ apart to avoid duplicates. The edit distance threshold varies depending on the task and window length. But it is recommended that $\theta$ is no bigger than half of the minimum window length.

\subsection{Contrast Visual Reasoning Patterns}

The subsequences are regarded as visual reasoning patterns that fulfill a certain goal. A contrast visual reasoning pattern is a subsequence that occurs much more frequently in one viewer group than the other. The contrast in occurrence signifies the uniqueness in the visual activity of this group and reveals the tacit visual reasoning processes. By converting the AOI sequence into a set of subsequences, the collection of scanpaths is similar to a transaction database and the contrast subsequences can be extracted using contrast mining [Dong and Bailey 2012].

We first define the frequency of a subsequence $S_{i, j}$ in a collection of $N$ scanpaths $\mathbb{S}=S^{1}, S^{2}, \cdots, S^{N}$ as support:

$$
\operatorname{supp}\left(S_{i, j}, \mathbb{S}\right)=\frac{\left|\left\{S^{k}: D_{x y}^{i j} \leq \theta, S_{x, y} \in S^{k}\right\}\right|}{N}
$$

which is the percentage of scanpaths in the collection that contains a similar subsequence to $S_{i, j}$.

With two groups of sequence collections $\mathbb{S}^{1}, \mathbb{S}^{2}$, the support difference of a subsequence $S_{i, j}$ is defined as growth:

$\operatorname{grow}\left(S_{i, j}, \mathbb{S}^{1}\right)=\left\{\begin{array}{cl}\min _{S_{x, y}\left(\frac{\operatorname{supp}\left(S_{i, j}, \mathbb{S}^{1}\right)}{\operatorname{supp}\left(S_{x, y}, \mathbb{S}^{2}\right)}\right),} & \exists S_{x, y} \in \mathbb{S}^{2}, D_{x y}^{i j} \leq \theta \\ \inf , & \forall S_{x, y} \in \mathbb{S}^{2}, D_{x y}^{i j}>\theta\end{array}\right.$

i.e. the minimum ratio of the support of $S_{i, j}$ in $\mathbb{S}^{1}$ and the support of its similar counterpart $S_{x, y}$ in $\mathbb{S}^{2}$.

Finally, pattern $S_{i, j}$ is a contrast visual reasoning pattern of $\mathbb{S}^{1}$ if both of its growth and support exceed the user defined thresholds. The set of contrast visual reasoning patterns of $\mathbb{S}$ is defined as $\mathbb{C}=$ $\left\{S_{i, j} \in \mathbb{S} \mid \operatorname{supp}\left(S_{i, j}, \mathbb{S}\right) \geq \rho, \operatorname{grow}\left(S_{i, j}, \mathbb{S}\right) \geq \epsilon\right\}$, where $\rho$ and $\epsilon$ are user-defined thresholds for support and growth. The result patterns are the AOI transitions that exhibited much more frequently in one group than in the other group. The choice of threshold is dependent on the visual task. Normally, the support is larger than 0.1 and the growth is larger than 1.5. The complexity for finding contrast patterns is $O\left(M^{2} N^{2}\right)$, where $M$ is the number of subsequences per scanpath and $N$ is the number of scanpaths per group.

\subsection{Pattern Distance and Hierarchical Clustering}

After the mining process, a group of scanpaths is represented by a set of contrast visual reasoning patterns $\mathbb{C}$. Some of the patterns in $\mathbb{C}$ might still be similar enough to be considered as the same visual process for a same goal. We use hierarchical clustering to find natural clusters of the patterns to produce explainable results.

Two distance measurements based on the sequence (subsequence string) and location (fixation locations in the subsequence) representations are proposed for different application scenarios. The two representations, with their emphasis on gaze sequence or location, are suitable for different applications. They also help investigate the importance of AOI orders for certain visual tasks. The distances also consider the difference between growth rates since they signify the uniqueness of the patterns. The distance function between two patterns using sequence representation is:

$$
\begin{gathered}
\operatorname{dist}_{\text {seq }}\left(S_{i, j}, S_{x, y}\right)=D_{x y}^{i j} * w_{\text {grow }} \\
w_{\operatorname{grow}}=\left(1+\left|\operatorname{ngrow}\left(S_{i, j}\right)-\operatorname{ngrow}\left(S_{x, y}\right)\right|\right) \\
\operatorname{ngrow}\left(S_{i, j}\right)=\frac{2}{1+e^{\left(1-\operatorname{grow}\left(S_{i, j}, \$\right)\right)}}-1
\end{gathered}
$$

where $w_{\text {grow }}$ is the weight calculated from growth difference, and $\operatorname{ngrow}\left(S_{i, j}\right)$ in $w_{\text {grow }}$ is the normalized growth of $S_{i, j}$. 
The location representation adopts the concept of bag-of-words vector to capture the frequency of AOI visits. We denote the location representation of $S_{i, j}$ as $V_{i, j}=\left[v_{1}, v_{2}, v_{3} \cdots\right]$, where $v_{p}=\mid\left\{s_{q}=\right.$ $\left.l_{p}: s_{q} \in S_{i, j}, l_{p} \in L\right\} \mid$. The distance between two patterns using location representation is based on cosine distance of the vectors:

$$
\operatorname{dist}_{\text {loc }}\left(S_{i, j}, S_{x, y}\right)=\left(1-\frac{V_{i, j} \cdot V_{x, y}}{\left\|V_{i, j}\right\|_{2}\left\|V_{x, y}\right\|_{2}}\right) * w_{\text {grow }}
$$

We then perform hierarchical clustering on both sequence and location representations using the two distance functions respectively to form dendrograms of the patterns. Average pair-wise distance (UPGMA) is applied to calculate the distance between clusters when forming hierarchies. The hierarchy of the clusters represents groups of visual activities at different levels of abstraction.

Using the same average pair-wise distance, silhouette score [Rousseeuw 1987] is applied to evaluate the clusters. The silhouette score reflects the consistency within clusters. The silhouette score, number of clusters, and the number of patterns per cluster serve as indications for the consistency and complexity in visual reasoning processes. The domain experts can quickly assess the variation within each group with these quantities.

\section{EXPERIMENT RESULTS AND DISCUSSION}

\subsection{Experiment Setting}

An image understanding and reasoning experiment was conducted with nine radiology images and questions. Two examples with marked AOIs (yellow polygons) are shown in Figure 1 and 3. There are 40.8 AOIs per image on average. The tasks represent a continuum from low-level image features judgement through high-level evaluation of the medical conditions. The nine questions can be grouped into three categories. Category 1 consists of images 1-3, and the questions range from asking image modality (e.g., CT, X-ray, MRIs) to basic visual elements. The second category includes images 4-8 and requires more anatomical knowledge. One question, as an example, is "What body part does this image demonstrate". Category 3, which uses image 9 and asks "What problem(s) do you think this patient has", requires extensive knowledge and experience.

A total number of 39 radiographers, 15 experts and 24 novices, participated and generated 646 scanpaths with two viewing sessions per subject, per image. With no constraint on viewing time, the scanpaths averaged 17.3 seconds in duration. The eye tracker was adapted from the ITU Gaze Tracker [San Agustin et al. 2010] which reached between $0.5^{\circ}-1^{\circ}$ of accuracy at 30 or $60 \mathrm{~Hz}$ sampling rate. The experiment was approved by the University of Missouri Health Science Institutional Review Board (IRB \#1172279).

Since most of the areas on the images are annotated with an AOI label, the raw fixation coordinates are mapped to the closest AOI. Fixations with a long dwell time (> $150 \mathrm{~ms}$ ) are segmented into $150 \mathrm{~ms}$ bins. The sequences are processed to find contrast patterns with minimum length of 4 and maximum length of $1 / 4$ of the whole scanpath. The thresholds are set to $\theta=2, \rho=0.2$ and $\epsilon=2$.

\subsection{Eye Movement Difference Quantification}

The purpose of silhouette score and number of clusters is to provide a quantifiable measurement for the consistency in the visual strategies for different tasks. Category 1 tasks asked about local

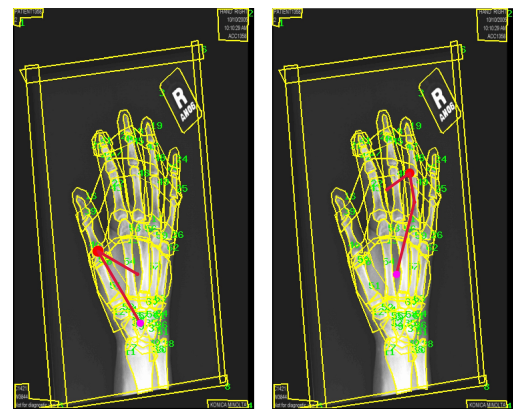

Figure 1: Typical contrast visual reasoning patterns of question 6. The experts' pattern (left) shows focused attention on the thumb. Novices (right) focus more on other four fingers.

image features that are not tied to any specific AOI. This is highlighted by the number of patterns and clusters. In question 3, the experts exhibited 131 different patterns, and these patterns could only be clustered at the highest levels of granularity. The novices, likewise, demonstrated a wide range of patterns (81) with little similarity. As the required semantic knowledge increased, the numbers of patterns decreased and the numbers of patterns per cluster increased. For example, in question 6, experts exhibited 47 patterns in 12 clusters, while this trend was not observed in novices.

The comparison of the scores between sequence and location representation can directly indicate the emphasis in different visual strategies. Questions 2, 5, 7, 8 for both groups and question 3 and 6 for the novice group have a much higher (3 to 10 times) number of patterns per cluster for sequence representation than location representation. This difference indicates that the visual reasoning processes differ mainly in the order of AOI visits while fixation locations are more consistent. Similarly, the silhouette scores for location representation are higher than sequence representation in nearly all cases. However, only experts in question 1, novices in question 2 and both groups in question 8 have a higher silhouette score for sequence representation. These quantified differences highlight the visual reasoning processes in certain questions.

To show the general effect of task difficulty and expertise, ANOVA tests were conducted using the silhouette score of each pattern with both sequence and location representations. Results show significant effect on task difficulty for both representations (sequence: $\mathrm{F}(8,929)=16.367, \mathrm{p}<.001$; location: $\mathrm{F}(8,929)=13.894, \mathrm{p}<.001)$, meaning that the visual strategies for some questions are more random than other questions. Such differences in silhouette scores correspond to the nature of the questions. Expertise level does not have a significant effect on location representations $(\mathrm{F}(1,929)=2.598$, $\mathrm{p}=0.107)$, while nearly having a significant effect on sequence representations $(\mathrm{F}(1,929)=3.317, \mathrm{p}=0.069)$. This result suggests the consistency in the order of fixation changes more drastically than location with the growth of expertise.

\subsection{Contrast Pattern Understanding}

Although expertise does not have a significant effect on overall silhouette scores, the patterns do show development through experience in some cases. For example, question 6 asks the radiographer 

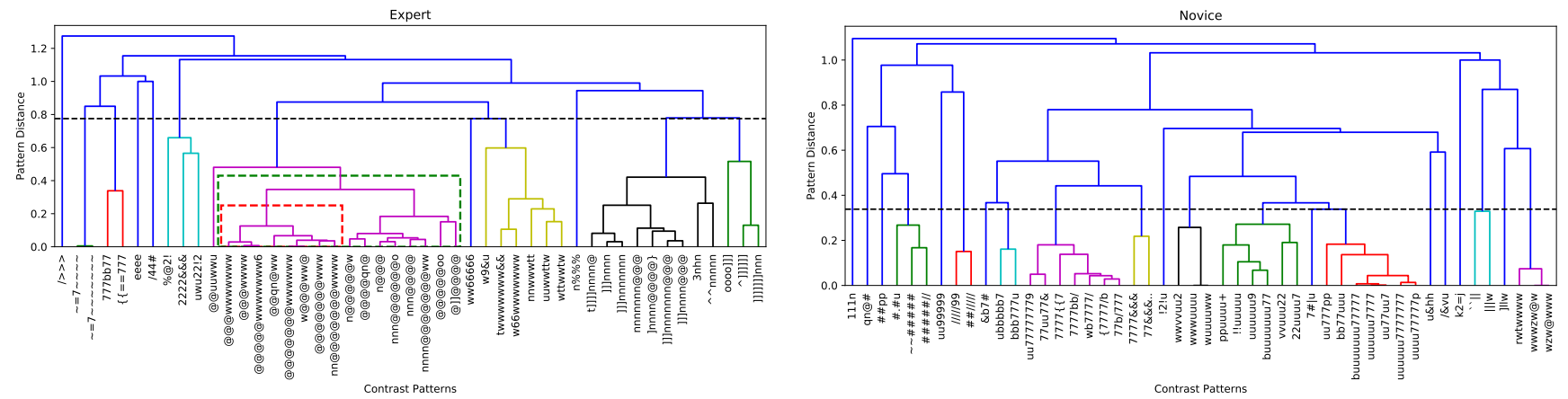

Figure 2: Dendrograms of contrast visual reasoning patterns for question 6 using location representation between expert and novice groups. The dash line marks the height with maximum silhouette score which forms the clusters marked by the colors.
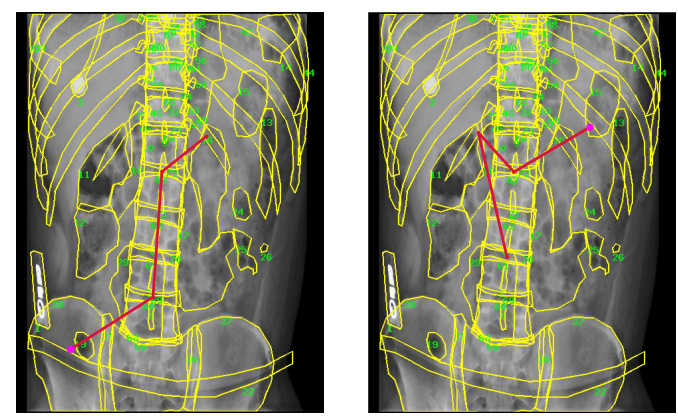

Figure 3: Typical visual patterns from expert (left) and novice (right) group for question 8.

to determine the position of the patient in relationship to the image receptor. In response to this question, expertise differences were seen in the numbers of clusters identified as shown in Figure 2, with experts exhibiting 12 clusters, in contrast to novices, who exhibited 21 clusters. Expertise differences were also seen in the average number of patterns per cluster (4.3 for experts and 2.4 for novices). This demonstrates that the AOI location and visiting order when determining the projection of an image become more consistent with the development of expertise. Still, some patterns were shared by both groups. For example, sequences where the radiographer evaluated the openness of the joint space of the interphalangeal joint (character "=" and "/" in the pattern string) and assessed the degree of concavity of the shafts of the phalanges ("b", "7" or "\{") was common in both groups. This indicates that this visual behavior is fundamental. However, the experts exhibited several sequences that focused on the 1st digit (thumb, "@") where the novices rarely fixated (example in Figure 1). The novices, on the contrary, fixated on irrelevant manufacturer's label ("l"), which is ignored by experts. The thumb is part of an important reasoning hierarchy shown by the dashed squares in Figure 2. At a lower reasoning level (red square), the common pattern ("@w") checks degrees of separation of metacarpal (palm) bones. Including the neighbor clusters, which examine the open joint spaces between carpal (wrist) bones ("n"), the higher leveled patterns (green square) allow the experts to determine the wrist position and hand projection.
Question 8 evaluated of the positioning of an image of the abdomen. The experts only exhibited 8 contrast patterns, which consistently scan the alignment of the spinous process with the spinal bodies and correlate that information with the shape of the pelvic wing and the distance from the spine to the ribs (example in left panel of Figure 3). In contrast, the novices exhibited 33 patterns, with the majority of those focusing on the pelvis. The representative patterns for novices also tended to travel horizontally or diagonally (right panel in Figure 3), where the experts consistently traveled in a vertical orientation. In this comparison, the experts have learned that the spine is the best indicator of a correctly positioned abdomen, where the novices are looking at numerous structures that are not as valuable to the evaluation of patient positioning.

\section{CONCLUSION AND FUTURE WORK}

The experiments show that the proposed method demonstrates its capability of finding frequent and contrasting visual activities between different viewer groups. The result patterns are intuitive and interpretable since they relate directly to the AOIs. Two different representations with emphases on the whole subsequence or only the AOI locations allow flexibility for different applications. The silhouette scores for the two representations are helpful when determining the difference in visual strategies for further examinations. The quantities may prove to be a useful tool in evaluating the development of visual knowledge expertise in visually-oriented fields, as they provide direct measurement of the visual behaviors. The different levels of visual reasoning abstraction with hierarchical clustering highlight the critical patterns for in-depth understanding of the reasoning structure of visual tasks.

The current method is planned be expanded with a custom distance matrix between the AOIs when calculating subsequence similarity to better capture the semantic differences. Additionally, the bottom-up visual saliency will be incorporated to associate image contents with gaze activities during the visual processes.

\section{ACKNOWLEDGMENTS}

We thank Dr. Hongfei Cao for initial experiment design and data collection. The project was partially supported by the National Science Foundation under grant number IIS-0812515. 


\section{REFERENCES}

Tobias Appel, Christian Scharinger, Peter Gerjets, and Enkelejda Kasneci. 2018. Crosssubject workload classification using pupil-related measures. In Proceedings of the 2018 ACM Symposium on Eye Tracking Research \& Applications - ETRA '18. ACM Press, New York, New York, USA, 1-8. https://doi.org/10.1145/3204493.3204531

Ali Borji, Dicky N. Sihite, and Laurent Itti. 2013. Quantitative Analysis of Human-Model Agreement in Visual Saliency Modeling: A Comparative Study. IEEE Transactions on Image Processing 22, 1 (jan 2013), 55-69. https://doi.org/10.1109/TIP.2012.2210727

Hongfei Cao, Yu Li, Carla M Allen, Michael A Phinney, and Chi-Ren Shyu. 2016. Visual Reasoning Indexing and Retrieval Using In-Memory Computing. International fournal of Semantic Computing 10, 03 (2016), 299-322. https://doi.org/10.1142/ S1793351X16400110

Siyuan Chen and Julien Epps. 2014. Using Task-Induced Pupil Diameter and Blink Rate to Infer Cognitive Load. Human-Computer Interaction 29, 4 (jul 2014), 390-413. https://doi.org/10.1080/07370024.2014.892428

Tim Chuk, Antoni B. Chan, and Janet H. Hsiao. 2014. Understanding eye movements in face recognition using hidden Markov models. Fournal of Vision 14, 11 (sep 2014), 8-8. https://doi.org/10.1167/14.11.8

Tim Chuk, Antoni B. Chan, and Janet H. Hsiao. 2017. Is having similar eye movement patterns during face learning and recognition beneficial for recognition performance? Evidence from hidden Markov modeling. Vision Research 141 (dec 2017), 204-216. https://doi.org/10.1016/J.VISRES.2017.03.010

Filipe Cristino, Sebastiaan Mathôt, Jan Theeuwes, and Iain D. Gilchrist. 2010. ScanMatch: A novel method for comparing fixation sequences. Behavior Research Methods 42, 3 (aug 2010), 692-700. https://doi.org/10.3758/BRM.42.3.692

Guozhu Dong and James Bailey. 2012. Contrast Data Mining: Concepts, Algorithms, and Applications (1st ed.). Chapman \& Hall/CRC.

Sukru Eraslan, Yeliz Yesilada, and Simon Harper. 2014. Identifying Patterns in Eyetracking Scanpaths in Terms of Visual Elements of Web Pages. Springer, Cham, 163-180. https://doi.org/10.1007/978-3-319-08245-5_10

Andreas Gegenfurtner, Erno Lehtinen, and Roger Säljö. 2011. Expertise Differences in the Comprehension of Visualizations: a Meta-Analysis of Eye-Tracking Research in Professional Domains. Educational Psychology Review 23, 4 (dec 2011), 523-552. https://doi.org/10.1007/s10648-011-9174-7

Joseph H. Goldberg and Jonathan I. Helfman. 2010. Scanpath clustering and aggregation In Proceedings of the 2010 Symposium on Eye-Tracking Research \& Applications ETRA '10. ACM Press, New York, New York, USA, 227. https://doi.org/10.1145/ 1743666.1743721

Michael J. Haass, Laura E. Matzen, Karin M. Butler, and Mika Armenta. 2016. A new method for categorizing scanpaths from eye tracking data. In Proceedings of the Ninth Biennial ACM Symposium on Eye Tracking Research \& Applications - ETRA '16. ACM Press, New York, New York, USA, 35-38. https://doi.org/10.1145/2857491. 2857503

Halszka Jarodzka, Kenneth Holmqvist, and Marcus Nyström. 2010. A vector-based, multidimensional scanpath similarity measure. In Proceedings of the 2010 Symposium on Eye-Tracking Research \& Applications - ETRA '10. ACM Press, New York, New York, USA, 211. https://doi.org/10.1145/1743666.1743718

Thomas C. Kübler, Enkelejda Kasneci, and Wolfgang Rosenstiel. 2014. SubsMatch: scanpath similarity in dynamic scenes based on subsequence frequencies. In Proceedings of the Symposium on Eye Tracking Research and Applications - ETRA '14. ACM Press, New York, New York, USA, 319-322. https://doi.org/10.1145/2578153.2578206

Thomas C. Kübler, Colleen Rothe, Ulrich Schiefer, Wolfgang Rosenstiel, and Enkelejda Kasneci. 2017. SubsMatch 2.0: Scanpath comparison and classification based on subsequence frequencies. Behavior Research Methods 49, 3 (jun 2017), 1048-1064. https://doi.org/10.3758/s13428-016-0765-6

Junpeng Lao, Sébastien Miellet, Cyril Pernet, Nayla Sokhn, and Roberto Caldara. 2017 iMap4: An open source toolbox for the statistical fixation mapping of eye movement data with linear mixed modeling. Behavior Research Methods 49, 2 (apr 2017), 559575. https://doi.org/10.3758/s13428-016-0737-x

Olivier Le Meur and Antoine Coutrot. 2016. Introducing context-dependent and spatially-variant viewing biases in saccadic models. Vision Research 121 (apr 2016), 72-84. https://doi.org/10.1016/J.VISRES.2016.01.005

Marcus Mast and Michael Burmester. 2011. Exposing repetitive scanning in eye movement sequences with t-pattern detection. In Proceedings IADIS International conference interfaces and human computer interaction (IHCI). 137-145. http://www.iadisportal.org/digital-library/ exposing-repetitive-scanning-in-eye-movement-sequences-with-t-pattern-detection

Mark Mills, Andrew Hollingworth, Stefan Van der Stigchel, Lesa Hoffman, and Michael D. Dodd. 2011. Examining the influence of task set on eye movements and fixations. Journal of Vision 11, 8 (jul 2011), 17-17. https://doi.org/10.1167/11.8.17 arXiv:NIHMS150003

Hamed R. Tavakoli, Adham Atyabi, Antti Rantanen, Seppo J. Laukka, Samia NeftiMeziani, and Janne Heikkilä. 2015. Predicting the Valence of a Scene from Observers' Eye Movements. PLOS ONE 10, 9 (sep 2015), e0138198. https://doi.org/10.1371/ journal.pone. 0138198

Kari-Jouko Räihä. 2010. Some Applications of String Algorithms in Human-Computer Interaction. Springer, Berlin, Heidelberg, 196-209. https://doi.org/10.1007/
978-3-642-12476-1 14

George E. Raptis, Christina Katsini, Marios Belk, Christos Fidas, George Samaras, and Nikolaos Avouris. 2017. Using Eye Gaze Data and Visual Activities to Infer Human Cognitive Styles. In Proceedings of the 25th Conference on User Modeling, Adaptation and Personalization - UMAP '17. ACM Press, New York, New York, USA, 164-173. https://doi.org/10.1145/3079628.3079690

Peter J. Rousseeuw. 1987. Silhouettes: A graphical aid to the interpretation and validation of cluster analysis. 7. Comput. Appl. Math. 20 (nov 1987), 53-65. https://doi.org/10.1016/0377-0427(87)90125-7

Javier San Agustin, Henrik Skovsgaard, Emilie Mollenbach, Maria Barret, Martin Tall, Dan Witzner Hansen, and John Paulin Hansen. 2010. Evaluation of a low-cost open-source gaze tracker. In Proceedings of the 2010 Symposium on Eye-Tracking Research \& Applications - ETRA '10. ACM Press, New York, New York, USA, 77. https://doi.org/10.1145/1743666.1743685

Dmitriy Shin, Mikhail Kovalenko, Ilker Ersoy, Yu Li, Donald Doll, Chi-Ren Shyu, and Richard Hammer. 2017. PathEdEx - Uncovering High-explanatory Visual Diagnostics Heuristics Using Digital Pathology and Multiscale Gaze Data. Fournal of Pathology Informatics 8 (jul 2017), 29. https://doi.org/10.4103/jpi.jpi 29_17

Jaana Simola, Jarkko Salojärvi, and Ilpo Kojo. 2008. Using hidden Markov model to uncover processing states from eye movements in information search tasks. Cognitive Systems Research 9, 4 (oct 2008), 237-251. https://doi.org/10.1016/J. COGSYS.2008.01.002

Julia M. West, Anne R. Haake, Evelyn P. Rozanski, and Keith S. Karn. 2006. eyePatterns: software for identifying patterns and similarities across fixation sequences. In Proceedings of the 2006 symposium on Eye tracking research \& applications - ETRA '06. ACM Press, New York, New York, USA, 149. https://doi.org/10.1145/1117309. 1117360 